\title{
AUDET, Louis-Philippe, Histoire de l'enseignement au Québec. Montréal, Holt, Rinehart et Winston, 1971. Vol. 1 : (1608-1840), xv-432 p.; vol. 2 : (1840-1971), xii-496 p. Ill. Index, bilbliographies. \$24.95.
}

\section{Marcel Lajeunesse}

Volume 26, numéro 3, décembre 1972

URI : https://id.erudit.org/iderudit/303195ar

DOI : https://doi.org/10.7202/303195ar

Aller au sommaire du numéro

Éditeur(s)

Institut d'histoire de l'Amérique française

ISSN

0035-2357 (imprimé)

1492-1383 (numérique)

Découvrir la revue

Citer ce compte rendu

Lajeunesse, M. (1972). Compte rendu de [AUDET, Louis-Philippe, Histoire de l'enseignement au Québec. Montréal, Holt, Rinehart et Winston, 1971. Vol. 1 : (1608-1840), xv-432 p.; vol. 2 : (1840-1971), xii-496 p. Ill. Index, bibliographies. \$24.95.] Revue d'histoire de l'Amérique française, 26(3), 427-432.

https://doi.org/10.7202/303195ar d'utilisation que vous pouvez consulter en ligne. 


\section{COMPTES RENDUS}

AUDET, Louis-Philippe, Histoire de l'enseignement au Québec. Montréal, Holt, Rinehart et Winston, 1971. Vol. 1: (16081840), XV-432 p.; vol. 2: (1840-1971), XII-496 p. Ill. Index, bibliographies. $\$ 24.95$.

Depuis son étude sur Le frère Marie-Victorin: ses idées pédagogiques (1942), Louis-Philippe Audet a accumulé les articles dans les Mémoires de la Société royale du Canaaa, les Cahiers des Dix, la Revue d'histoire de l'Amérique française, la Revue de l'Université Laval, le Dictionnaire biographique du Canada. Il a aussi publié, jusqu'à maintenant, six volumes de son Système scolaire de la province de Québec, en plus de collaborer à de nombreux ouvrages collectifs, notamment avec J. Donald Wilson et Robert M. Stamp, ainsi qu'avec Armand Gauthier. Depuis longtemps, on souhaitait que Louis-Philippe Audet nous donne une vue d'ensemble de l'histoire de l'éducation québécoise dont il avait analysé tant d'aspects. La vaste synthèse de près de 1,000 pages, couvrant plus de trois siècles et demi d'histoire, soit de 1608 à 1971, qu'il nous présente vient à point nommé.

L'ouvrage de monsieur Audet recèle de grandes richesses. C'est une véritable encyclopédie de l'enseignement québécois, toutes les lois scolaires sont expliquées, décortiquées, le fonctionnement de l'appareil scolaire démêlé, le rôle des hommes politiques, des administrateurs scolaires et des ecclésiastiques interprété, la vie des enseignants commentée. C'est aussi une introduction à l'histoire des idées pédagogiques du monde atlantique. De plus, l'on peut faire de ce volume un survol de l'histoire du Québec.

Dans l'avant-propos, l'auteur relate le cheminement de cette synthèse et expose clairement ses objectifs:

1) étudier le passé pour comprendre le présent;

2) le faire avec objectivité sans passion et sans tomber dans l'apologétique;

3) intégrer l'étude de l'organisation scolaire dans le jeu complexe des forces politiques, économiques et sociales du Québec depuis trois siècles et demi (I: XIII-XIV).

Ces objectifs sont ceux que visait Antoine Prost dans son excellent ouvrage sur l'enseignement en France. ${ }^{1}$ De même, monsieur Audet a utilisé une présentation similaire à celle de l'étude de Prost: chacun des chapitres est suivi de documents justificatifs, courts, mais bien choisis, et d'une bibliographie. D'utile qu'elle est pour tout l'ouvrage, cette orientation bibliographique devient extrêmement précieuse pour guider le lecteur et le chercheur

1 Antoine Prost, Histoire de l'enseignement en France, 1800-1967 (Paris, Armand Colin, 1968), 524 p. Collection U.

RHAF, vol. 26, no 3 (décembre 1972) 
à travers la documentation, fournie mais disparate, de la période contemporaine.

L'auteur a évité le piège d'une relation de législation scolaire, de l'histoire traditionnelle de l'organisation scolaire, sans lien avec l'histoire globale d'une collectivité. Les institutions scolaires sont des institutions sociales (I: 86; II: 465). Il a mis à profit le sage conseil que le sociologue Durkheim donnait aux historiens de l'enseignement voilà près de quarante ans.

L'auteur a écrit deux cent dix pages sur l'éducation en NouvelleFrance. Tout historien connaît la difficulté d'apporter des éléments nouveaux sur cette période depuis les minutieuses recherches d'Amédée Gosselin. Depuis la publication de son Système scolaire, v. 2, qui reprenait, en fait, Groulx et Gosselin, Audet a poursuivi ses recherches sur les "petites écoles", en essayant de donner des précisions quantitatives sur celles-ci, les instituteurs et les élèves, principalement à l'extérieur des villes de Québec et de Montréal. Il a approfondi sa connaissance des institutions scolaires de la métropole et des pédagogies qui y avaient cours, en exploitant habilement les travaux de de Rochemonteix, de de Dainville, d'Allain, de Snyders, de Pontet. Les recherches effectuées par l'historien français Pontet ${ }^{2}$ cernent l'œuvre pédagogique et religieuse de Jean-Baptiste de la Salle et éclairent l'œuvre scolaire des frères des Ecoles chrétiennes, des frères Charon et des Sulpiciens. L'incidence de ces travaux sur la connaissance en NouvelleFrance est considérable, et il faut remercier l'auteur de nous les avoir fait connaître.

Monsieur Audet a, également, beaucoup profité du portrait plus authentique de la Nouvelle-France qu'ont tracé les historiens au cours des dernières décennies. Il a fait son profit des études de Hamelin sur l'histoire économique et sociale, de Henripin sur la population, de R.-L. Séguin sur la civilisation matérielle, de Frégault sur l'Eglise et les finances canadiennes, de Trudel sur un essai de synthèse de cette période.

Le chapitre sur la "Formation des structures scolaires françaises" (I: 20-42), bien que fort intéressant et heureusement court, apparait comme un hors-d'œuvre: sans doute aurait-il fallu voir parmi les quelques lectures sur "Le Moyen Age - les Universités", le petit volume de Jacques LeGoff (Les intellectuels du Moyen Age).

2 Yves Pontet, "La Compagnie de Saint-Sulpice et les petites écoles de Montréal au XVIIe siècle (1657-1700)", Bulletin du Comité des études, Compagnie de Saint-Sulpice, Paris, (avril-juin 1961): 164-184. - "L'auteur de l'Escole paroissiale (Démia) et quelques usages de son temps (1654)", Bulletin de la Société des bibliophiles de Guyenne (Bordeaux, janvier-juin 1963), $28 \mathrm{p}$. - - "Les voux des Frères Charon, Hospitaliersenseignants", Revue d'histoire de l'Eglise de France (1963) : 19-46. "Une institution franco-canadienne au XVIII ${ }^{\circ}$ siècle: les écoles populaires de garçons à Montréal", Revue d'histoire ecclésiastique (Louvain), LIX, no 2 (1964) : 52-88; no $3(1964): 437-488$. - Le XVII siècle et les origines lassalliennes (Rennes, Imprimeries réunies, 1970), 2 v. Thèse de doctorat ès lettres. 
La connaissance de la pédagogie française au XVII ${ }^{e}$ siècle, avec ses influences catholiques (de la Salle, Démia), jansénistes (Port-Royal des Champs), protestants (Bacon, Comenius, Locke), est essentielle pour comprendre les mentalités et les réalisations en Nouvelle-France:
... l'état de l'enseignement élémentaire et de l'enseignement se- condaire, en France, durant le $X V I I^{e}$ siècle, aura pour le Canada une importance capitale, puisque la moitié environ des 10,000 colons qui s'établirent en Nouvelle-France, durant le régime français, viendront entre 1608 et $1700 . .$. Mais cet héritage s'est également incarné dans tous ces maîtres d'écoles - Récollets, Jésuites, Sulpiciens, ecclésiastiques du clergé séculier, Frères Charon, Ursulines, Sours de l'Hôpital-Général de Québec, Saurs de la Congrégation de Notre-Dame, notaires, maîtres ambulants - ... (I: 44)

Nous sommes bien d'accord avec l'auteur pour donner à Jean-Baptiste de la Salle une place de choix sur le plan de la pensée pédagogique et sur le plan du rayonnement scolaire de même que sur l'ambivalence de l'idée d'instruction populaire chez les philosophes et les encyclopédistes. Mais monsieur Audet nous semble bien sévère envers Jean-Jacques Rousseau ...

Sur le Collège des Jésuites de Québec, fondé en 1635, un an avant celui d'Harvard, sur le modèle des collèges jésuites de France, nous avons des précisions nouvelles, résultats des recherches de l'auteur et de celles du père Lucien Campeau sur les commencements de collège.

Reprenant l'expression de Félix Ponteil: "L'Eglise est maîtresse de l'Ecole" sous l'ancien régime, Audet nous montre bien qu'au XVII et au $\mathrm{XVIII}^{\mathrm{e}}$ siècles, tant dans la métropole que dans les colonies, le rôle de l'Etat consistait à surveiller, à diriger et à régulariser. Le clergé (séculier et régulier) fondait des écoles et le gouvernement du Roi de France l'encourageait de son autorité, de ses conseils et de ses deniers par des dotations ou des subsides. On se rend compte que le jugement célèbre de Garneau sur l'éducation en Nouvelle-France est un exemple de l'histoire écrite à la lumière du présent, à une époque (1845) où l'instruction était passablement dégradée au Canada-Est; à une époque aussi où les pays atlantiques se donnaient des systèmes d'éducation cohérents et vigoureux.

Le lecteur québécois apprendra beaucoup en lisant le chapitre sur la "Formation des structures scolaires anglo-saxonnes". Les noms des pédagogues Vivès, Ascham, Mulcaster, Hoole, Bill, Stow, lui seront plus familiers et rejoindront ceux, mieux connus, de Bacon, Locke et Lancaster. Il verra que la vieille tradition pédagogique de l'Ecosse a vivifié les idées pédagogiques du monde britannique à l'époque moderne. Il constatera que le vaste mouvement d'émigration britannique en Amérique du Nord, à l'époque moderne, fut également une transplantation de traditions européennes dans lesquelles les écoles et les collèges s'intégrèrent aisément. Les "grammar schools" et les "colleges" (Harvard, William and Mary, etc.) de la Nouvelle-Angleterre étaient conçus sur les modèles métropolitains. 
Même s'il est souvent téméraire de tenter de déceler les influences anglo-saxonnes sur l'enseignement québécois dans le siècle qui suit la Conquête, l'auteur n'a aucune difficulté à en identifier un certain nombre: le projet d'université de 1789 , la loi scolaire créant l'Institution royale pour l'avancement des sciences (1801), l'université McGill (1821), le passage de Joseph Lancaster dans la colonie et la promotion de l'enseignement mutuel (1818, 1829-1830), l'enquête d'Arthur Buller (1838) et les Lettres sur l'éducation élémentaire et pratique de Mondelet (1840), la loi scolaire de 1841 et celles qui suivirent dans les décennies 1840 et 1850 , l'échec du projet de loi d'Hector Langevin destiné à donner à la minorité anglo-saxonne du Québec un surcroît de protection (1866) et le vote de l'article 93 de l'AANB, grâce à l'influence et à la détermination de Galt (1867).

La synthèse, allant de la Conquête à nos jours, couvre 660 pages. L'auteur a employé fort judicieusement la coupure naturelle en histoire de l'enseignement du Québec venant de l'abolition du ministère de l'Instruction publique en 1875: 356 pages de texte pour $1760-1875$, et 304 pages pour 1875-1971.

Pour la période 1760-1875, l'auteur a fait un usage constant des documents d'archives (Institution royale, enquête Buller, enquête Sicotte, abolition du ministère de l'Instruction publique, etc.), des rapports des surintendants et leurs bilans, à partir de 1842 (pour les administrations Me lleur et Chauveau). En ce qui a trait à l'histoire générale de cette période, l'auteur a eu recours, principalement, aux volumes de Ouellet et Hamelin, Chapais, Wade, Sylvain et Labarrère. Quant à la période 1875-1971, l'auteur fait grand usage des documents officiels (rapports du Département de l'Instruction publique, rapports de commissions d'enquête, etc.). Pour l'histoire générale de cette période il utilise abondamment l'histoire monumentale de Rumilly.

Décidément, monsieur Audet n'aime pas la controverse autour du problème de la Conquête. Au v. I: 272-73, l'auteur explicite sa pensée sur la Conquête et ses interprètes, de même que sur les relations entre les deux solitudes. Ses propos, et la citation de Grotius (I: 279) révèlent l'influence de Mgr Maheux.

Le portrait de Carleton-Dorchester dessiné par l'auteur touche à l'image d'Epinal. Sans doute faut-il voir, dans son désir de concilier les classes dirigeantes canadiennes, une certaine conception aristocratique de la société et le souci de freiner la demande d'une Chambre d'Assemblée.

Les études très fouillées de monsieur Audet sur l'Institution royale sont connues. Expliquant la genèse de cette institution qui se voulait un véritable ministère de l'instruction publique, pour les anglophones surtout, l'auteur montre que les Loyalistes qui avaient vécu, en Nouvelle-Ang.eterre, près de collèges dynamiques et de qualité, se firent au Bas-Canada, les apôtres d'un véritable systıme d'éducation (I: 342-343). Le différend EgliseAssemblée lors de la loi des Ecoles de Syndics (1829) et de la loi des Notables (1832) n'est pas souligné.

La carrière de Jean-Baptiste Meilleur à la surintendance des écoles fut une véritable tragédie personnelle. Monsieur Audet exprime dans les pages 
qu'il lui consacre une réelle sympathie pour le courage du docteur Meilleur de même que pour l'étendue et la difficulté de sa charge. Ce n'était pas la tâche de l'auteur de fournir une étude spécialisée sur la guerre des Eteignoirs; mais une histoire sociale de cet événement, à partir des greffes des notaires sans doute, serait nécessaire pour renouveler le sujet, pour en éclairer la genèse, le déroulement, de même que les protagonistes.

Nous croyons que l'auteur, en utilisant l'étude Houle préparée par la Commission Parent, met le point final à un long débat historico-juridique en affirmant que, si l'on se rapporte aux lois scolaires du début de la décennie 1840 qui sont à l'origine des commissions scolaires, "il est certain que celles-ci n'avaient aucun caractère confessionnel et les écoles qu'elles administraient étaient "communes", c'est-à-dire ouvertes à tous les enfants d'âge scolaire de la municipalité sans distinction de religion. Cependant, la loi acceptait de diverses manières, notamment en reconnaissant le droit de dissidence, que l'école puisse être confessionnelle" (II: 366).

Il est intéressant de noter que les raisons qui ont motivé la création d'un ministère de l'Instruction publique en 1867 étaient semblables à celles qui ont établi un ministère de l'Education en 1964: le surintendant, étant un fonctionnaire soumis à un ministre responsable, qui n'a pas la capacité de présenter, à l'Assemblée, les lois et les budgets de l'instruction publique, est privé de la responsabilité ministérielle (II: 102). Par ailleurs, la quasi-unanimité des historiens québécois de la fin du $\mathrm{XIX}^{\mathrm{e}}$ siècle et de la première moitié du $\mathrm{XX}^{\mathbf{e}}$ siècle sur la suppression de ce ministère, est révélatrice de leur idéologie. On admettait qu'il fallait un haut fonctionnaire (surintendant) pour s'occuper de l'administration; encore que certains ( $\mathrm{Mgr}$ Moreau) regrettaient que ce fût un laïque, même si ce laïque était un homme de confiance et qu'il était, en quelque sorte, l'otage du Conseil de l'Instruction publique.

Monsieur Audet souligne, en ce qui a trait aux communautés religieuses françaises, deux faits importants: d'abord la venue de nombreuses communautés à partir de 1837 (frères des Ecoles chrétiennes), possédant des traditions pédagogiques; ensuite, l'apport éducatif et idéologique des religieux chassés de France par les lois Combes au début du $\mathrm{XX}^{\mathrm{e}}$ siècle.

Pour ce qui est de la seconde moitié du $\mathrm{XIX}^{\mathrm{e}}$ siècle et le $\mathrm{XX}^{\mathrm{e}}$, on ne peut que déplorer le peu de recherches qui se font sur l'enseignement supérieur et sur l'enseignement technique. Une étude qui serait le pendant de la thèse de Stamp, The Campaign for Technical Education in Ontario, 18761914 (1970) répondrait à beaucoup de questions.

Il y eut, au Québec, une prolifération d'institutions de formation pédagogique dans la première moitié du $\mathrm{XX}^{\mathrm{e}}$ siècle: "l'histoire de l'évolution des écoles normales catholiques au Québec ressemble un peu à un mauvais rêve" (II: 317). Au début des années 1960, sur les 150 institutions du genre au Canada, le Québec en comptait plus de 120, si l'on exclut les écoles universitaires de pédagogie et les écoles normales spéciales.

Pour la période récente, monsieur Audet nous livre une synthèse fort utile des commissions d'enquête et de nombreux comités d'études qui tou- 
chent l'éducation et qu'il était nécessaire de sortir de l'ombre qui commence à les envelopper. Ces commissions et ces comités montrent le fourmillement de la pensée et de l'activité en éducation au début de la décennie 1960. Ces commissions sont la Commission Parent (éducation, 1961), Bélanger (fiscalité, 1963), Bouchard (commerce du livre, 1963) Laurendeau-Dunton (bilinguisme et biculturalisme, 1963), Rioux (enseignement des arts, 1966), et Deschênes (université, 1969). Pour les comités, nous trouvons le Comité d'étude sur l'enseignement agricole et agronomique (Régis, 1961), sur l'enseignement technique et professionnel (Tremblay, 1961), en éducation des adultes (Ryan, 1962), sur les loisirs, l'éducation physique et les sports (Bélisle, 1962), sur l'enseignement dans les écoles d'architecture de Montréal et de Québec (Lamontagne, 1963) et sur l'enseignement professionnel agricole (Lettre, 1963).

En lisant Audet, on se convainc que la formation des maitres n'a pas reçu, dans la forme scolaire, toute l'attention qu'elle exigeait. On s'en est occupé tardivement - en 1966 - et l'action gouvernementale fut assez peu dynamique et cohérente. Nous pouvons, de plus, nous poser de sérieuses questions sur l'importance que devait prendre, à l'Université du Québec, la formation des maîtres. L'auteur fait, à la fin de son ouvrage, de sérieuses réserves sur les effets du syndicalisme des enseignants.

Les idées exprimées dans la conclusion sont élevées, mais on peut se demander si l'auteur a raison d'être aussi pessimiste devant l'évolution de l'éducation actuelle. Il est permis, enfin, de ne pas partager entièrement sa notion "du maître qui marque ses disciples" (II: 474).

L'histoire du Québec s'est donc enrichie, en 1971, d'une grande œuvre de synthèse et d'un ouvrage de référence essentiel. A la qualité intrinsèque de ces deux volumes fort bien écrits, malgré quelques fautes de langue ou de typographie, il faut ajouter la beauté et le raffinement de la présentation tant intérieure qu'extérieure. La maison Holt, Rinehart et Winston en a fait une édition de prestige. D'ailleurs son prix élevé (\$24.95) le met hors du pouvoir d'achat des étudiants en général. Nous sommes devant l'un de ces cas d'édition en fonction des institutions. Il ne faudra pas se surprendre de voir cet ouvrage dans toutes les véritables bibliothèques d'ici et de l'étranger. 